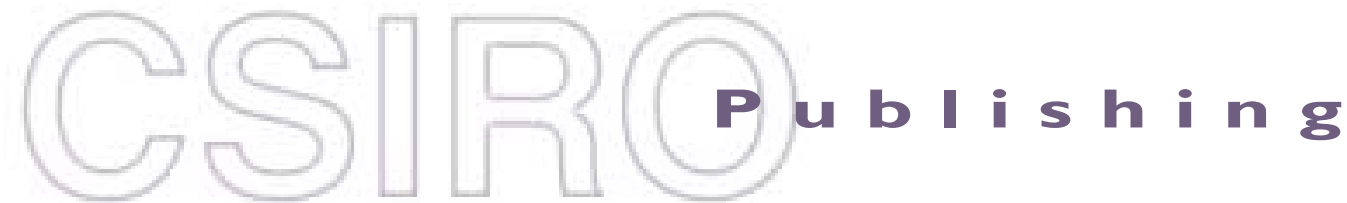

\section{Publications of the Astronomical Society of Australia}

Volume 19, 2002

(C) Astronomical Society of Australia 2002

An international journal of astronomy and astrophysics

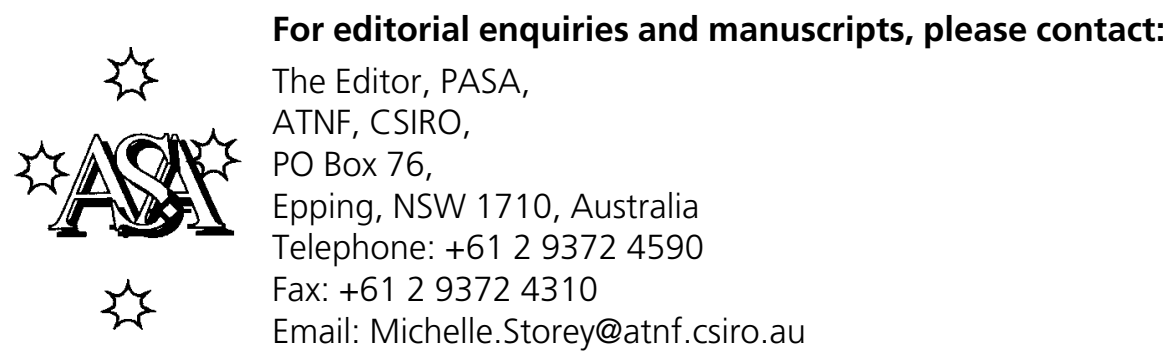

For general enquiries and subscriptions, please contact: CSIRO Publishing PO Box 1139 (150 Oxford St)

Collingwood, Vic. 3066, Australia

Telephone: +6139662 7666

Fax: +61 396627555

Email: publishing.pasa@csiro.au

C S I RO

PUBLISHING Published by CSIRO Publishing

for the Astronomical Society of Australia

www.publish.csiro.au/journals/pasa 


\title{
Circular Polarisation in AGN
}

\author{
Jean-Pierre Macquart* \\ School of Physics, University of Sydney, NSW 2006, Australia \\ jpm@physics.usyd.edu.au \\ Received 2001 August 20, accepted 2001 October 2
}

\begin{abstract}
We discuss the constraints that recent observations place on circular polarisation in AGN. In many sources the circular polarisation is variable on short timescales, indicating that it originates in compact regions of the sources. The best prospects for gleaning further information about circular polarisation are high resolution VLBI and scintillation 'imaging' which probe source structure on milliarcsecond and microarcsecond scales respectively.
\end{abstract}

Keywords: polarisation - galaxies: active - galaxies: nuclei - scattering

\section{Introduction}

The mean degree of circular polarisation (CP) in AGN is typically less than $1 \%$, but its origin is not understood. Although the linear polarisation observed in AGN is accepted as evidence of synchrotron emission, the physical significance - hence the correct interpretation of the CP in AGN is not clear. This is largely because, until now, the observations were of insufficient quality to constrain the properties of the CP.

New data from the VLBA and the Australia Telescope Compact Array (ATCA) have enabled a reassessment of the origin of CP. The high angular resolution of the VLBA gives it a decisive advantage over most telescopes in circular polarimetry. Since the magnitude of the CP is usually small and occurs primarily in compact regions, high resolution is necessary to mitigate the effects of beam depolarisation. The VLBA has also been able to, for the first time, determine the location of a circularly polarised region in an AGN relative to its core and jets.

Paradoxically, the ATCA, with its modest angular resolution, has made equally important contributions to our understanding of CP. Its advantage is the demonstrated ability to measure the degree of CP in sources to better than $0.01 \%$. It is particularly useful when the angular resolution of the instrument is irrelevant because there is some other means of obtaining high angular resolution information about the source, usually from source variability. Its ability to do high precision, short timescale monitoring of intraday variable (IDV) radio sources, coupled with an understanding of interstellar scintillation, allows it to achieve microarcsecond resolution polarimetry.

We outline in Section 2 the various possible origins of the $\mathrm{CP}$ and in Section 3 the constraints placed upon these mechanisms by the observations. Various other sources that exhibit CP are discussed in Section 4. The conclusions are presented in Section 5.

\footnotetext{
* Present address: Kapetyn Astronomical Institute, University of Groningen, The Netherlands.
}

\section{Origins of Circular Polarisation}

\subsection{Synchrotron Emission}

There is a small degree of $\mathrm{CP}$ associated with synchrotron emission. For an electron with Lorentz factor $\gamma$ gyrating around a field line at an angle $\theta$ to the line of sight the degree of CP observed is (Legg \& Westfold 1968)

$$
m_{c}=\frac{\cot \theta}{3}\left(\frac{v}{3 v_{H}}\right)^{-1 / 2} \approx \cot \theta / \gamma
$$

in an optically thin source, where $v_{H}=e B \sin \theta / m_{e}$ is the electron gyrofrequency. The degree of $\mathrm{CP}$ for an optically thick source remains similar; it is modified by a term of order unity dependent on the distribution of relativistic electrons, $N(\epsilon) \propto \epsilon^{-2 \alpha-1}$ (Melrose 1971). In a homogeneous source the sense of the $\mathrm{CP}$ is expected to reverse as the source becomes optically thick.

Equation (1) implies that high $\mathrm{CP}$ is associated with emission from particles with low Lorentz factors, $\gamma$, or emission nearly parallel to the magnetic field (i.e. small $\theta$ ). However, in both instances the power expected from synchrotron radiation is small. The brightness temperature of particles radiating with Lorentz factor $\gamma$ is limited by self-absorption to $T_{B} \lesssim(\gamma-1) m_{e} c^{2} / k \approx 0.6 \times$ $10^{10}(\gamma-1) \mathrm{K}$. However, bulk relativistic motion of the emitting region toward the observer can Doppler boost the radiation, permitting higher degrees of $\mathrm{CP}$ for a given observed brightness temperature.

The emission intensity in synchrotron radiation is proportional to $(\sin \theta)^{\alpha+1}$. This falls to zero for emission nearly parallel to the magnetic field lines (i.e. as $\theta \rightarrow 0$ ). Although a high degree of $\mathrm{CP}$ is expected from radiation parallel to the magnetic field, it is associated with low power emission. Furthermore, regions with small $\theta$ would be confined to a small volume of the source in any plausible model for the distribution of the magnetic field. The importance of magnetic field non-uniformity is implied by the fact that the degree of linear polarisation observed in AGN is at least an order of magnitude lower than the $\sim 70 \%$ expected due to synchrotron radiation. This suggests that 
the level of $\mathrm{CP}$ expected due to synchrotron radiation is at least an order of magnitude lower than that expected from equation (1).

The low levels of $\mathrm{CP}$ expected from this emission mechanism and the fact that its $m_{c} \propto v^{-1 / 2}$ dependence is generally not observed (see references in Saikia \& Salter 1998) suggest that synchrotron emission is not responsible for most of the CP observed in extragalactic sources at centimetre wavelengths.

\subsection{Cyclotron Emission}

Cyclotron emission refers to radiation from non-relativistic electrons (i.e. $\gamma-1 \ll 1$ ). If the electron orbit is edge-on to the line of sight then $100 \%$ linearly polarised radiation is observed and, if face-on, the radiation is fully circularly polarised. Elliptically polarised radiation is observed for intermediate orientations.

Cyclotron emission occurs at frequencies that are integer multiples of the cyclotron frequency, $v_{H}$. The power of the emission at the harmonics of the cyclotron frequency is proportional to $\sin \theta$, and thus falls to zero as $\theta \rightarrow 0$. The radiative power in these harmonics therefore falls to zero as the emission becomes more circularly polarised. The radiative power is non-zero for $\theta=0$ only for the emission at the fundamental frequency, and this is the only radiation which may be, in principle, fully circularly polarised.

The brightness temperature of the emission in the rest frame of the emitting particles is low: $T_{B} \sim 0.6 \times 10^{10} \mathrm{~K}$. Unless the cyclotron emission is highly Doppler boosted any $\mathrm{CP}$ associated with it is easily diluted by emission from brighter, synchrotron emitting regions.

\subsection{Coherent Emission Mechanisms}

Coherent emission processes have the potential to account for CP in AGN. The polarisation of pulsar radiation suggests that this is plausible. Pulsar radiation must be due to coherent emission, since the brightness temperature of the radiation is in the range $T_{B} \approx 10^{22}-10^{26} \mathrm{~K}$. The emission is also observed to be highly polarised (e.g. Manchester, Taylor, \& Huguenin 1975), however it is possible that the $\mathrm{CP}$ in pulsars is not intrinsic to the radiation mechanism at all, but instead arises due to propagation effects in the pulsar magnetosphere.

It is difficult to make specific comments about the polarisation properties of any coherent emission that may occur in AGN since (i) there is no preferred mechanism for the coherent emission, (ii) the polarisation properties of most of the various mechanisms have not been considered in detail, and (iii) it is unknown whether the physical conditions necessary for the coherent emission would also lead to depolarisation of the escaping radiation. This last point is relevant because any coherent emission observed in AGN is likely to emanate from many localised patches emitting simultaneously (e.g. Melrose 1991; Benford 1992), which would lead to averaging out of the polarisation properties of each individual patch. Nonetheless, some specific mechanisms predict no CP (Windsor \& Kellogg 1974; Benford 1984).
Coherent mechanisms are not required to explain high brightness temperature emission in IDV sources (see Melrose, these proceedings), and they do not seem to be explicitly required to explain the few per cent levels of $\mathrm{CP}$ seen in these sources either. Furthermore, if these processes do operate in AGN it is not obvious that they would dominate incoherent synchrotron emission or propagation induced polarisation effects.

\subsection{Faraday Conversion in a Relativistic Plasma}

Propagation can convert CP from linear polarisation in a process analogous to Faraday rotation, called generalised Faraday rotation or Faraday repolarisation (Sazonov 1969; Pacholczyk 1973; Jones \& O’Dell 1977).

The transfer of polarised radiation in a birefringent plasma may be understood in terms of the Poincaré sphere (Figure 1). A magnetised homogeneous plasma has two mutually orthogonal natural wave modes, with some difference in wavenumber between the two modes, $\Delta k \neq 0$. The wave modes of a given birefringent medium define a diagonal through the origin and opposite ends on the surface of the Poincaré sphere. Propagation through the plasma induces a phase difference $L \Delta k$ between the two orthogonally polarised wavefields which results in a rotation of the polarisation vector around the Poincaré sphere at constant latitude around the axis defined by the natural modes.

In a magnetised relativistic plasma the natural modes of the medium are elliptically polarised. Propagation of radiation through such a plasma converts linearly polarised

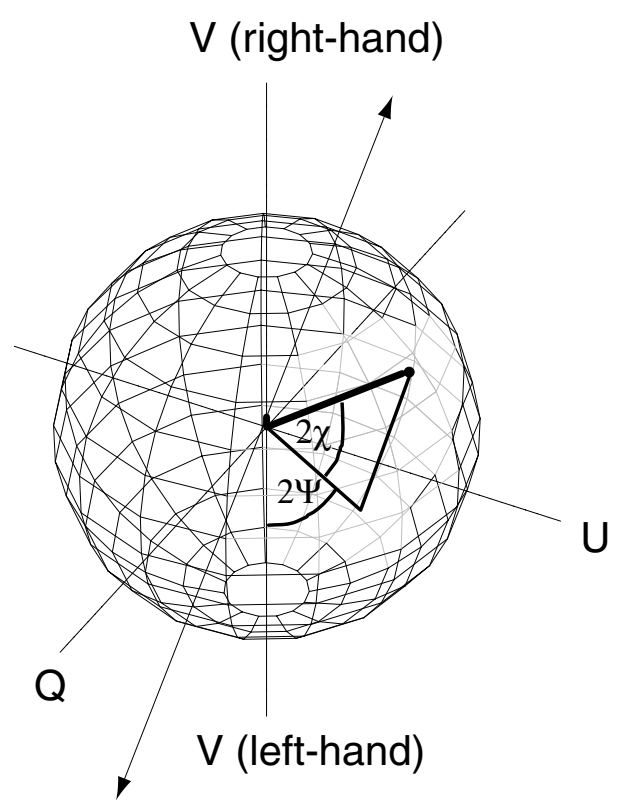

Figure 1 The Poincaré sphere may be used to represent the polarisation state of radiation. Elliptically polarised radiation is mapped from the Stokes parameters $\mathcal{P} / I=[Q, U, V] / I$ to the coordinates $[\cos (2 \chi) \cos (2 \Psi), \cos (2 \chi) \sin (2 \Psi), \sin (2 \chi)]$. The arrowed diagonal depicts a case in which natural modes of a medium are elliptical. Generalised Faraday rotation in such a medium corresponds to rotation of the polarisation vector at constant latitude about this axis. 
radiation into circularly polarised radiation as follows (Kennett \& Melrose 1998):

$$
\begin{aligned}
V(v)= & U_{0}(v) \sin \left(\lambda^{3} \mathrm{RRM}\right), \\
\operatorname{RRM}= & 3 \times 10^{4}\left(\frac{L}{1 \mathrm{pc}}\right) \\
& \times\left\langle\mathcal{E}_{L}\left(\frac{n_{r}}{1 \mathrm{~cm}^{-3}}\right)\left(\frac{B}{1 \mathrm{G}}\right)^{2} \sin ^{2} \theta\right\rangle \mathrm{rad} \mathrm{m}^{-3},
\end{aligned}
$$

where the relativistic rotation measure, RRM, depends upon the density of relativistic particles, $n_{r}$, the path length, $L$, the magnetic field, $B$, and the minimum Lorentz factor of the pairs, $\mathcal{E}_{L}$. This effect operates only when the direction of the incident linear polarisation is at an oblique angle to the projection of the magnetic field on the plane orthogonal to the ray direction. The axes used to define the Stokes parameters may be chosen such that synchrotron emission has $Q \neq 0, U=0$. With this choice, the effect occurs only if the incident radiation has $U_{0} \neq 0$, requiring either Faraday rotation or that it originates from a region of the source where the magnetic field is in a different direction to that in the region where the polarisation conversion takes place.

A characteristic of this model is a strong frequency dependence on the sign of $V$. If RRM is high enough to produce appreciable amounts of $\mathrm{CP}$ at high frequency, this model predicts rapid changes in its handedness at low frequency. In a relativistic plasma, the position angle of the radiation defined by the $U-V$ plane rotates $\propto \lambda^{3}$.

However, the structure of the source can have important consequences for the frequency dependence of the $\mathrm{CP}$. The Blandford-Königl jet is a particular and relevant instance of this (Blandford \& Königl 1979). Its largely self-similar structure can ensure that, at each frequency, the $\mathrm{CP}$ is of constant sign and comparable magnitude. This is because the emission height of the $\mathrm{CP}$ observed along the jet varies as a function of frequency.

One specific configuration for producing moderate (a few per cent) CP with consistent sign (Ruszkowski \& Begelman, submitted) involves a plasma with a weak uniform magnetic field and a highly spatially variable random magnetic field. The random component, with high rotativity per unit length, can generate moderate degrees of CP. Because this model relies on a highly spatially inhomogeneous magnetic field, one expects that the $\mathrm{CP}$, as observed on the plane of the sky, is also highly spatially inhomogeneous. The viability of this model can be constrained from scintillation observations, as described in Section 3.

\subsection{Scintillation-induced Circular Polarisation}

Another model that relies upon a highly inhomogeneous medium involves scintillation-induced CP (Macquart \& Melrose 2000a,b). This is produced when even unpolarised radiation propagates through a region with nonuniform $n_{e} B$ (related to the Faraday rotation per unit length). A transverse gradient in this quantity leads to different ray bending for the right- and left-hand circularly polarised radiation. Since the wavefronts are also rippled due to the phase delays caused by the inhomogeneity, as occurs in scintillation, transverse displacement of the two wavefronts can induce CP. The difference in bending angles between the two senses of polarisation is small but the radiation propagates over a large distance, so that the displacement between the two wavefronts, and hence the $\mathrm{CP}$, can be appreciable. This theory may provide an explanation for the variable $\mathrm{CP}$ observed at $\mathrm{cm}$ wavelengths in some AGN and in Sgr A*.

There is no preferred handedness for the sense of the $\mathrm{CP}$ produced by this mechanism: although the mean square $\mathrm{CP}$ is non-zero, the mean $\mathrm{CP}$, averaged over a sufficiently long time span, is zero. One expects sign changes in the $\mathrm{CP}$ on a timescale associated with the scintillation pattern. This is not observed. However, this model needs to be extended to remove some of the constraining assumptions on which it is based. The most notable of these is the assumption that the inhomogeneities occur on a thin screen. The relaxation of this assumption to take into account the extended nature of the medium would affect both the magnitude of the effect and the timescale on which sign changes are predicted to occur.

\section{Observational Properties}

In this section we concentrate on the connection between the $\mathrm{CP}$ and its variability. Rayner (these proceedings) contains a further discussion on the origins of the $\mathrm{CP}$ in AGN.

The $\mathrm{CP}$ in AGN was known to be variable almost twenty years ago (e.g. Weiler \& de Pater 1983; Komesaroff et al. 1984). In their monitoring of a sample of AGN at $5 \mathrm{GHz}$, Komesaroff et al. (1984) observed several general features which have been borne out by more accurate measurements:

- Changes in CP occur with only small accompanying changes in the total intensity. Interpreting these in terms of an intrinsic change in the source due to, say, the emergence of a new component, the authors argued this implied degrees of CP typically $~ 5 \%$ in the polarised subcomponents of these sources. (These arguments also apply if the variability is due to scintillation.)

- The timescale of variability in $\mathrm{CP}$ is shorter than that of the linear polarisation, which in turn is shorter than that in the total intensity.

- The magnitude of the fractional variability in the $\mathrm{CP}$ usually exceeds that in the linear polarisation, which exceeds that in the total intensity.

Comparison with recent ATCA and VLBA observations shows that many sources retain the same handedness of $\mathrm{CP}$ over decade-long time scales. However, early measurements indicated that some sources do change sign: Komesaroff et al. (1984) noted sources 2 out of 14 on the $\sim 100$ day timescale that the observations were sensitive to. The reality of these sign changes has not been pursued with recent observations. 
The CP appears to occur in compact regions. VLBI observations by Homan \& Wardle (1999) and Homan, Attridge, \& Wardle (2001) support the general conclusion that the $\mathrm{CP}$ is associated with the compact regions near the cores of AGN.

It is not clear why some sources exhibit high degrees of $\mathrm{CP}$. There appears to be no connection between the degree of $\mathrm{CP}$ and the linear polarisation (Rayner, Norris, \& Sault 2000). VLBI suggests this may be due to Faraday depolarisation of the linearly polarised radiation (e.g. Homan et al. 2001). There is, however, a tentative connection between high CP and IDV AGN. Many of these sources exhibit strong and variable $\mathrm{CP}$; these sources include PKS 1519-273 (Macquart et al. 2000), PKS 0404-385 and PKS 1144-379. In each case the degree of CP implied by comparing the changes in Stokes $V$ and $I$ is a few per cent ${ }^{1}$. The last two sources exhibit $\mathrm{CP}$ variability on shorter timescales than that observed in the total intensity, suggesting that it emanates from an even more compact region that does the emission responsible for the bulk of the unpolarised variability.

Are the degrees of CP inferred in IDV sources atypical? The possibility that many other sources have high CP in regions so compact that they appear beam depolarised even on VLBI scales remains to be investigated.

\subsection{The Significance of CP Variability}

The prevalence of $\mathrm{CP}$ variability in AGN suggests that it occurs predominantly in compact regions. One might expect that it should vary more rapidly than $I$ because it can also change sign. However, this is unlikely to be the correct explanation for the short timescale. Both Stokes $Q$ and $U$ can also change sign in the same manner as the $\mathrm{CP}$, but they are not, in general, observed to vary as quickly. The rarity of sign reversals in the $\mathrm{CP}$ also argues against this, especially when compared to the large position angle changes observed in linear polarisation in the same sources.

The fact that the simultaneous total flux density and polarised flux density variations in IDV sources are different is often used as evidence against the viability of scintillation in these sources. While scintillation affects all four Stokes parameters of the propagating radiation identically $^{2}$, the structure of the scintillating source complicates the issue.

A simple source model comprised of two compact (scintillating) components illustrates this. Suppose one component is faint but highly (say 10\%) circularly polarised, while the other is strong but unpolarised. If the angular separation between the sources is comparable to the angular scale of the scintillations $\left(\sim \theta_{\mathrm{F}}=r_{\mathrm{F}} / D\right.$ for weak scintillations) then the fluctuations due to each of the components are uncorrelated. Furthermore, because the fluctuations in $\mathrm{CP}$ come from one component while

\footnotetext{
${ }^{1}$ The mean degree of $\mathrm{CP}$ in these three sources ranges $\approx 0.2-0.8 \%$.

${ }^{2}$ This is strictly only true if the medium is unmagnetised, but it holds to a good approximation for scintillations in our Galaxy's ISM.
}

the majority of the unpolarised flux density variations originate from the other, the fluctuations in Stokes $V$ and $I$ show only a small correlation.

The nature of the correlation may change during the course of a year if the direction of the scintillation velocity changes. Such changes are possible because changes in Earth's velocity, due to its orbit about the Sun, can substantially alter the apparent scintillation velocity. If the two source components lie in a line parallel to the scintillation velocity one observes identical fluctuations in both components, but with a delay corresponding to the angular separation of the components relative to the scale of the scintillation pattern. If the velocity is orthogonal, one sees a loss of correlation, with a large decorrelation if the angular separation of the components is greater than the relevant scintillation decorrelation length divided by distance to screen (i.e. $\theta_{\mathrm{F}}$ in the weak scattering regime).

However, real sources are more complicated than two blobs. VLBI polarimetric imaging (e.g. Gabuzda, Pushkarev, \& Cawthorne 2000) shows a great richness of detail and structure in the linear polarisation of milliarcsecond components in AGN. If IDV sources exhibit the same richness of structure on microarcsecond scales that they do on VLBI scales it would be surprising that the polarisation lightcurves of IDV sources bear any resemblance at all to the fluctuations in total intensity! Indeed, many IDV sources show rich structure in polarisation lightcurves relative to the total intensity.

This suggests that attempts to model source variability in terms of a finite number of distinct components are missing the point. One instead ought to be talking about measuring the power spectrum of the brightness distribution in each of the four Stokes parameters.

It is possible to invert for the source structure in a rigorous manner if the variability is due to scintillation. Formally, the power spectrum of the observed fluctuations is the product of the scintillation power spectrum due to a point source, which is known or can be determined, and the power spectrum of the source angular brightness distribution; see Macquart \& Jauncey (2001 submitted) for more detail, and Figure 2 for an intuitive explanation of the effect of source structure on its lightcurve. This technique would be useful in testing the model advanced by Begelman for which one expects small-scale structure in the CP.

\section{Lessons from Other Sources}

$\operatorname{Sgr} A^{*}$

$\mathrm{CP}$ has been detected in $\mathrm{Sgr} \mathrm{A}^{*}$ from 1.4 to $8.6 \mathrm{GHz}$ with ATCA and the VLA (Bower, Falcke, \& Backer 1999; Sault \& Macquart 1999). The mean degree of CP is $0.3 \%$ but the $\mathrm{CP}$ varies on a timescale of $\sim 10$ days, much faster than the monthly to yearly variability observed in the total flux density at these frequencies. The origin of the variability is not well constrained; it could be intrinsic or due to scintillation of a compact component in the source. 
(i)

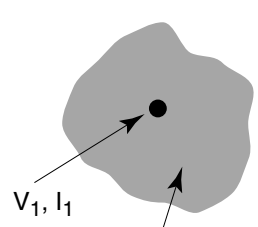

$\mathrm{I}_{0}$

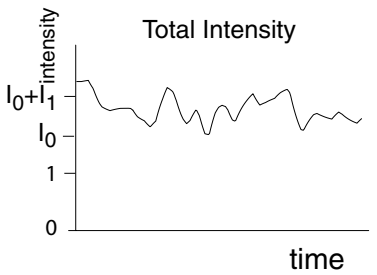

time

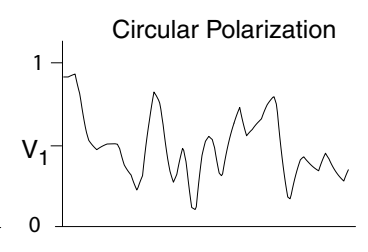

(ii)

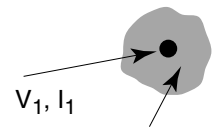

$\mathrm{I}_{0}$

(iii)

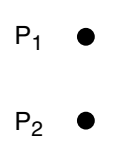

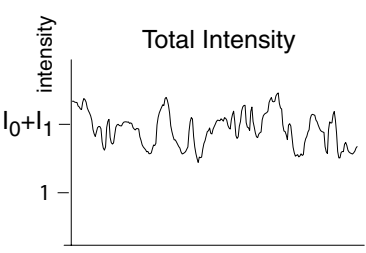

Polarization 1

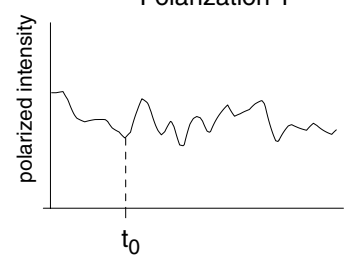

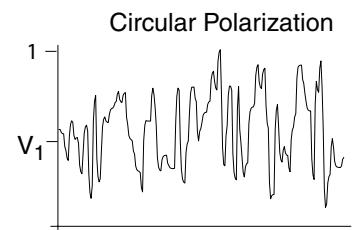

Polarization 2

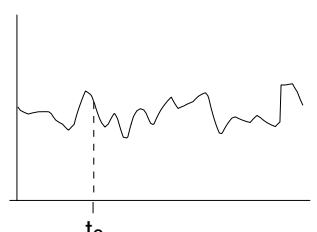

Figure 2 Typical lightcurves for common source configurations: (i) a polarised sub-source embedded in a source too large to scintillate, (ii) two scintillating sources, with the smaller component embedded in the larger, and (iii) two distinct polarised components.

The spectrum of the mean degree of $\mathrm{CP}$ is flat from 1.4 to $8.6 \mathrm{GHz}$ but the significance of this is complicated by the fact that one does not know the degree of $\mathrm{CP}$ in the polarised component. It appears that only a small portion of the source is polarised. Changes in the $\mathrm{CP}$ are accompanied by only small changes in the total intensity. If the variability in the $\mathrm{CP}$ is indeed associated with the variability of a specific component of the source, the 1.4 and $2.4 \mathrm{GHz}$ ATCA data suggest the presence of a subcomponent with an intrinsic $\mathrm{CP}$ of a few percent.

\section{Galactic Microquasars}

$\mathrm{CP}$ is observed in several Galactic microquasars. $\mathrm{CP}$ was first detected in SS433 by Fender et al. (2000) but the data were insufficient to determine the origin of the CP. While the total intensity emission and linearly polarised emission were unresolved, the $\mathrm{CP}$ was not. It was suggested that the $\mathrm{CP}$ originated in the inner parts of the system and that the actual polarisation of these regions was as high as $10 \%$.

CP has recently been discovered in GRO 1655-40 from ATCA archival data of the August 1994 outburst (J.-P. Macquart et al., in preparation). The time evolution of the CP was observed at frequencies from 1.4 to $9 \mathrm{GHz}$, and shows the $\mathrm{CP}$ changing sign during the early stages of the outburst, reaching a maximum and decaying over a period comparable to the decay of the total emission.

\section{Conclusions}

The advent of high precision circular polarimetry has enabled a reassessment of the $\mathrm{CP}$ in AGN. However, most of the outstanding questions twenty years ago regarding the origin of the CP in AGN still remain.

VLBI and variability (scintillation) measurements indicate the $\mathrm{CP}$ in AGN is associated with the emission near their cores, and that it occurs in very compact regions. Future measurements of the $\mathrm{CP}$ will therefore only be useful if the observations are capable of resolving this polarised emission.

The best means of obtaining this resolution at the centimetre wavelengths at which the $\mathrm{CP}$ is currently being detected is via scintillation 'imaging'. ATCA data indicate that a large proportion of IDV sources exhibit variable CP. Scintillation data can be used to extract information about the degree of the $\mathrm{CP}$ and its location relative to the linearly polarised and unpolarised emission.

High precision polarimetry over a larger range of frequencies would be useful in constraining the origin of the circularly polarised emission. However, its use may be limited. Complications due to source inhomogeneity and structure limit the degree to which one can use the spectral slope of the CP to constrain its origin.

\section{Acknowledgements}

It is a pleasure to thank Don Melrose, Dave Rayner, and Bob Sault for their advice and help. 


\section{References}

Benford, G. 1984, ApJ, 282, 154

Benford, G. 1992, ApJ, 391, L59

Blandford, R. D., \& Königl, A. 1979, ApJ, 232, 34

Bower, G. C., Falcke, H., \& Backer, D. C. 1999, ApJ, 523, L29

Fender, R., Rayner, D., Norris, R., Sault, R. J., \& Pooley, G. 2000, ApJ, 530, L29

Gabuzda, D. C., Pushkarev, A. B., \& Cawthorne, A. W. 2000, MNRAS, 319, 1109

Homan, D. C., \& Wardle, J. F. C. 1999, AJ, 118, 1942

Homan, D. C., Attridge, J. M., \& Wardle, J. F. C. 2001, ApJ, 556,113

Jones, T. W., \& O'Dell, S. L. 1977, ApJ, 215, 236

Kennett, M. P., \& Melrose, D. B. 1998, PASA, 15, 211

Komesaroff, M. M., Roberts, J. A., Milne, D. K., Rayner, P. T., \& Cooke, D. J. 1984, MNRAS, 208, 409

Legg, M. P. C., \& Westfold, K. C. 1968, ApJ, 154, 99

Macquart, J.-P., \& Melrose, D. B. 2000a, Phys. Rev. E, 62, 4177
Macquart, J.-P., \& Melrose, D. B. 2000b, ApJ, 545, 798

Macquart, J.-P., Kedziora-Chudczer, L., Rayner, D. P., \& Jauncey, D. L. 2000, ApJ, 538, 623

Manchester, R. N., Taylor, J. H., \& Huguenin, G. C. 1975, ApJ, 196,83

Melrose, D. B. 1971, Ap\&SS, 12, 172

Melrose, D. B. 1991, Ann. Rev. Astron. Astrophys., 29, 31

Pacholczyk, A. G. 1973, MNRAS, 163, 29P

Rayner, D. P., Norris, R. P., \& Sault, R. J. 2000, MNRAS, 319,484

Ruszkowski, M. \& Begelman, M. C. 2002, ApJ, submitted (astro$\mathrm{ph} / 0112090)$

Saikia, D. J., \& Salter, C. J. 1988, Ann. Rev. Astron. Astrophys., 26, 93

Sault, R. J., \& Macquart, J.-P. 1999, ApJ, 526, 85L

Sazonov, V. N. 1969, Zh. Eksper Teor. Fiz., 56, 1074

Weiler, K. W., \& de Pater, I. 1983, ApJ Supp., 52, 293

Windsor, R. A., \& Kellogg, P. J. 1974, ApJ, 190, 167 